\title{
Colloidal Stabilization of Calcium Carbonate Prenucleation Clusters with Silica
}

\author{
Matthias Kellermeier, Denis Gebauer, Emilio Melero-García, Markus Drechsler, \\ Yeshayahu Talmon, Lorenz Kienle, Helmut Cölfen, * Juan Manuel García-Ruiz, and \\ Werner Kunz*
}

Calcium carbonate precipitation proceeds via a complex multistage scenario involving neutral ion clusters as precursors and amorphous phases as intermediates, which finally transform to crystals. Although the existence of stable clusters in solution prior to nucleation has been demonstrated, the molecular mechanisms by which they precipitate are still obscure. Here, direct insight into the processes that drive the transformation of individual clusters into amorphous nanoparticles is provided by progressive colloidal stabilization of different transient states in silica-containing environments. Nucleation of calcium carbonate in the presence of silica can only take place via cluster aggregation at low $\mathrm{pH}$ values. At higher $\mathrm{pH}$, prenucleation clusters become colloidally stabilized and cannot aggregate. Nucleation through structural reorganization within the clusters is not observed under these conditions, indicating that this pathway is blocked by kinetic and/or thermodynamic means. The degree of stabilization against nucleation is found to be sufficient to allow for a dramatic enrichment of solutions with prenucleation clusters and enable their isolation into the dry state. This approach renders direct analyses of the clusters by conventional techniques possible and is thus likely to facilitate deeper insight into the chemistry and structure of these elusive species in the future.

\section{Introduction}

The basic principles behind nucleation and growth of crystals are still in the focus of scientific interest, even more than 70 years after the classical nucleation theory was introduced. ${ }^{[1,2]}$ There is increasing evidence that in biological and biomimetic mineralization, crystallization can occur through alternative, particle-based reaction channels, ${ }^{[3,4]}$ which may involve amorphous ${ }^{[5-9]}$ or liquid precursors. ${ }^{[10-14]}$ Calcium carbonate is an often chosen model system in this context due to its broad importance as an abundant mineral of biological ${ }^{[15]}$ as well as geological origin, ${ }^{[16]}$ related implications for the natural $\mathrm{CO}_{2}$ cycle, ${ }^{[17]}$ its use in many industrial applications, ${ }^{[18]}$ and the fact that it is one of the major sources of water hardness and thus responsible for scaling and incrustation. ${ }^{[19]}$

Recent work revealed that prior to nucleation of solid $\mathrm{CaCO}_{3}$, neutral ion clusters are formed as solutes even in undersaturated solutions. ${ }^{[20-22]}$ As opposed to classical theories, these so-called prenucleation clusters exist in thermodynamic equilibrium with the dissolved ions ${ }^{[20]}$ and were first imaged by Pouget et al. with the aid of cryogenic transmission electron microscopy (cryo-TEM). ${ }^{[23]}$ Further, computer simulations and re-evaluations of experimental data suggested that prenucleation clusters are in fact highly dynamic, liquidand chain-like polymers of calcium carbonate ion pairs. ${ }^{[24]}$ This structural form rationalizes the thermodynamic stability of the clusters on the basis of strong hydration in combination with a distinct entropic contribution originating from a high degree of disorder. Simulations further indicated that a transition of such polymeric species towards bulk-like structures requires a
Dr. M. Kellermeier, Dr. D. Gebauer, Prof. H. Cölfen Physical Chemistry

University of Konstanz

Universitätsstrasse 10, 78457 Konstanz, Germany

E-mail: helmut.coelfen@uni-konstanz.de

Dr. E. Melero-García, Prof. J. M. García-Ruiz

Laboratorio de Estudios Cristalográficos

IACT (CSIC-UGR), Avda. del Conocimiento s/n

P.T. Ciencias de la Salud, 18100 Armilla, Spain

Dr. M. Drechsler

Institute of Macromolecular Chemistry II

University of Bayreuth

Universitätsstrasse 30, 95440 Bayreuth, Germany
Prof. Y. Talmon

Department of Chemical Engineering

Technion-Israel Institute of Technology, Haifa 32000, Israel

Prof. L. Kienle

Technical Faculty

Christian-Albrechts-University Kiel

Kaiserstrasse 2, 24143 Kiel, Germany

Prof. W. Kunz

Institute of Physical and Theoretical Chemistry

University of Regensburg

Universitätsstrasse 31, 93053 Regensburg, Germany

E-mail: werner.kunz@chemie.uni-regensburg.de 
significant barrier to be overcome, ${ }^{[24]}$ which can be correlated to the activation energy associated to a nucleation event.

In light of the existence of stable solute precursors with relatively large volumes, it seems unlikely that statistical clustering of ions into un- or metastable nuclei-as envisaged in the classical picture-plays an important role in the nucleation of calcium carbonate. It rather appears as if prenucleation clusters are the relevant species in this context, ${ }^{[20]}$ and their transformation into a solid phase may proceed along one of the following two fundamental pathways (or a complex combination of both, depending on experimental conditions): i) structural rearrangement within prenucleation clusters towards metastable amorphous calcium carbonate (ACC) or direct conversion to crystalline modifications and ii) aggregation of the clusters into larger assemblies, which subsequently undergo phase transformation.

Although the factual height of the activation barrier associated to pathway (i) remains unknown, ${ }^{[24]}$ one may speculate that it is significant as compared to thermal energy, ${ }^{[22]}$ essentially because experimental observations support an aggregation-based mechanism (ii), which initially yields ACC nanoparticles with diameters in the range of $\approx 30 \mathrm{~nm} \cdot{ }^{[20,23]}$ That is, scenario (i) may be kinetically inhibited under the investigated conditions and require substantial amounts of time to occur, unless suitable "catalysts" like nucleation-promoting surfaces ${ }^{[25]}$ (i.e., the heterogeneous case) or certain specialized additives such as bio(macro)molecules are present. Moreover, there are fundamental questions regarding the energetics of nanoscale calcium carbonate, which might render pathway (i) thermodynamically improbable. For instance, it has been suggested that there is a crossover of the thermodynamic stabilities of distinct polymorphs at the nanoscale, ${ }^{[26]}$ and theoretical considerations indicate that ACC is indeed the stable phase for nanoparticles of calcium carbonate smaller than $4 \mathrm{~nm}$. ${ }^{[27]}$ This would clearly discriminate scenario (i) from a classical point of view-at least when it comes to the direct formation of crystalline modifications from prenucleation clusters-as long as there is no thermodynamic stabilization of nanosized crystalline particles provided (e.g., by additives or surfaces).

It is evident that differentiating between the two possible pathways by experimental means is a highly difficult task in pure systems. This is essentially due to the inherently low concentrations of the precursors at practical supersaturation levels, as well as the fact that corresponding processes might take place on very short timescales, such that detailed analyses become impossible and transient intermediates may simply be overlooked. In order to assess if nucleation of calcium carbonate can proceed via one or both of the pathways outlined above under a given set of conditions, and whether kinetic or thermodynamic constraints dominate nucleation, it appears necessary to prepare solutions enriched with prenucleation clusters and decelerate their transformation in the system. Herein, we show that addition of silica is an effective means to achieve this. The presented methodology can be tuned to either completely suppress nucleation of $\mathrm{CaCO}_{3}$ particles even at high supersaturation, or to progressively stabilize different transient states on the way to the solid phase. Our findings provide new insight into the mechanisms of nucleation in the presence of additives and demonstrate that colloidal stabilization of prenucleation clusters with silica can afford relatively concentrated cluster solutions, thus facilitating their characterization in situ. Beyond that, our approach even allows for an isolation of these species and hence represents a promising strategy towards detailed future analyses of their structure, which is urgently needed.

\section{Results and Discussion}

\subsection{Nanoclusters in Silica-Rich Solutions of Calcium Carbonate}

When solutions of calcium chloride and sodium carbonate (each $5 \mathrm{~mm}$ ) are mixed in the presence of moderate amounts of silica, instant precipitation takes place and spherical nanoparticles of ACC with diameters $\geq 30 \mathrm{~nm}$ are generated immediately. It has previously been demonstrated that these particles become stabilized in this case due to spontaneous deposition of a skin of amorphous silica all over their surface, which impedes energetically favored transformation to calcite. ${ }^{[28]}$ By contrast, above a certain threshold of silica concentration $(\approx 3000 \mathrm{ppm}$ at $\mathrm{pH} 11)$, a clear solution is obtained and nucleation seems to be suppressed. This notion is confirmed by cryo-TEM images of samples quenched shortly after mixing, which reveal that $\mathrm{CaCO}_{3}$ exists in the form of numerous nanoclusters, with apparent diameters typically ranging from 0.5 to $3 \mathrm{~nm}$ (Figure 1). From a comparison of the resulting size distribution with data reported in literature for neat $\mathrm{CaCO}_{3}$ solutions, ${ }^{[23]}$ we infer that these species are prenucleation clusters of calcium carbonate, as will be further rationalized below. Their occurrence in the samples is supported independently by analytical ultracentrifugation (AUC). The measured sedimentation coefficients $(s)$ can be subdivided into four relevant intervals (see Table S1 and Figure S1 in the Supporting Information), partly following a classification suggested by Pouget et al.:[23] values in the range of $0-0.5 \mathrm{~S}$ are assigned to ions, which were traced in all experiments. Species with $0.5<s<2$ were consistently found in samples containing silica, regardless of the presence of $\mathrm{CaCl}_{2}$ and/or $\mathrm{Na}_{2} \mathrm{CO}_{3}$, and are therefore interpreted as lowmolecular silicate oligomers (dimers, trimers, cyclic structures, etc.) commonly present in alkaline silica sols. ${ }^{[29]}$ Mixtures of $\mathrm{CaCl}_{2}, \mathrm{Na}_{2} \mathrm{CO}_{3}$ and silica contain a further population with $2<s<3.5$, likely corresponding to the multitude of individual nanoclusters observed in the cryo-TEM images. Repeatedly, a fourth component was identified $(>5 \mathrm{~S})$, which can be allocated to cluster aggregates in agreement with light and X-ray scattering data (see below). Interestingly, the sedimentation coefficients found for single clusters are somewhat higher than those reported previously $(1.5-3 \mathrm{~S}){ }^{[20,23]}$ This difference may originate from binding of oligomeric silica in the periphery of the clusters, as indicated also by dynamic light scattering (DLS) data (see Section 2.2). It is furthermore worth noting that species with $s$-values in the range of nanoclusters and cluster aggregates were detected also in reference samples containing silica and either $\mathrm{Na}_{2} \mathrm{CO}_{3}$ or $\mathrm{CaCl}_{2}$. Their origin is discussed in detail below (Section 2.5).

From the diffusion coefficients determined simultaneously by AUC, hydrodynamic diameters $\left(d_{\mathrm{H}}\right)$ were derived for the different species via the Stokes-Einstein equation (Supporting 

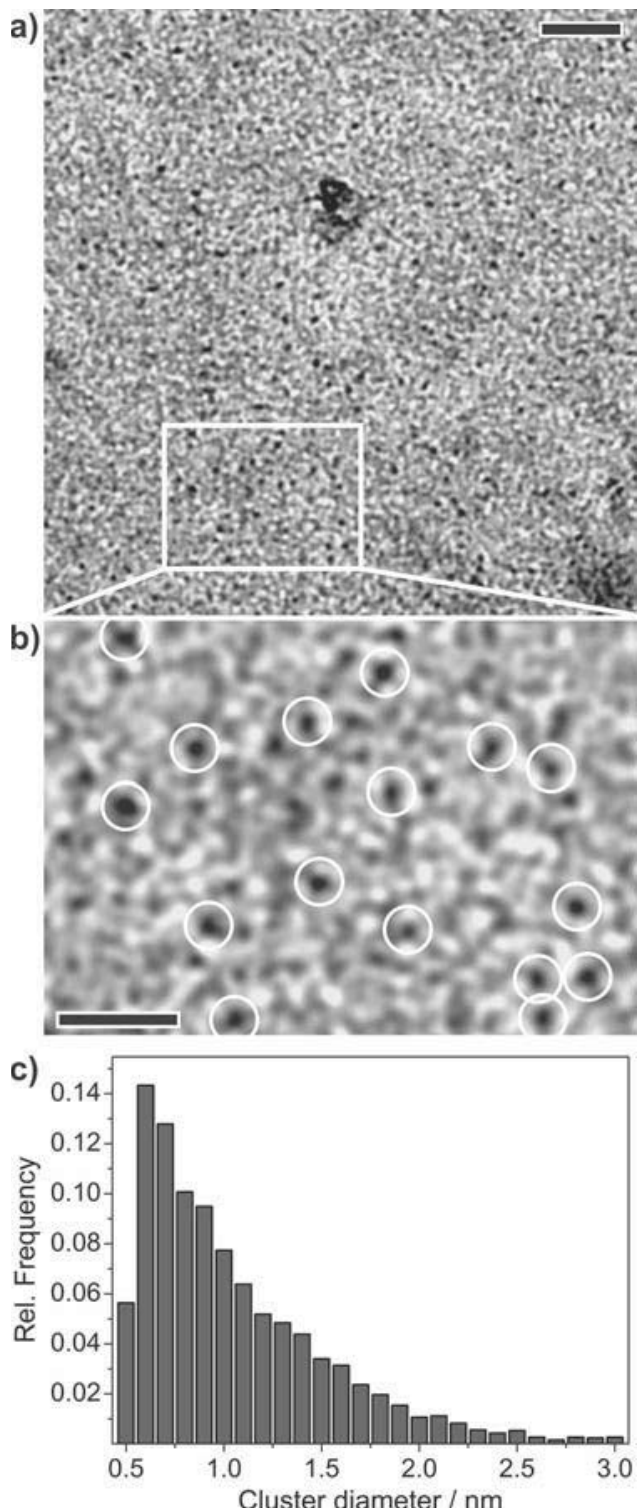

Figure 1. Nanoclusters in silica-rich supersaturated solutions of calcium carbonate. a) Cryo-TEM image of a sample containing $5 \mathrm{~mm} \mathrm{CaCO}_{3}$ and $3720 \mathrm{ppm} \mathrm{SiO}{ }_{2}$ at $\mathrm{pH}$ 11, quenched in liquid ethane 3 min after mixing reagents. Scale bar: $20 \mathrm{~nm}$. b) Enlargement of the area delimited by the white rectangle in (a), illustrating the presence of individual nanoclusters (highlighted by white circles). Scale bar: $10 \mathrm{~nm}$. c) Size distribution diagram derived from cryo-TEM image for individual cluster species. The apparent average cluster diameter is $1.1 \pm 0.6 \mathrm{~nm}$. Note that, due to the difficulty of interpreting electron contrast at the resolution limit of the technique, the determined values may not necessarily reflect the true size of the clusters. This would explain the observed discrepancies between diameters obtained from cryo-TEM images and the scattering data (cf. Figure 3 and 4 ).

Information, Table S2). Apparent sizes obtained for silica oligomers were found to vary between 0.7 and $1.7 \mathrm{~nm}$, while the diameters of clusters ranged from 2 to $6 \mathrm{~nm}$ (an overlap of these two populations is likely, though). Previous calculations for additive-free $\mathrm{CaCO}_{3}$ solutions gave an average value of about $2 \mathrm{~nm}$ for single clusters, ${ }^{[20]}$ which again suggests the
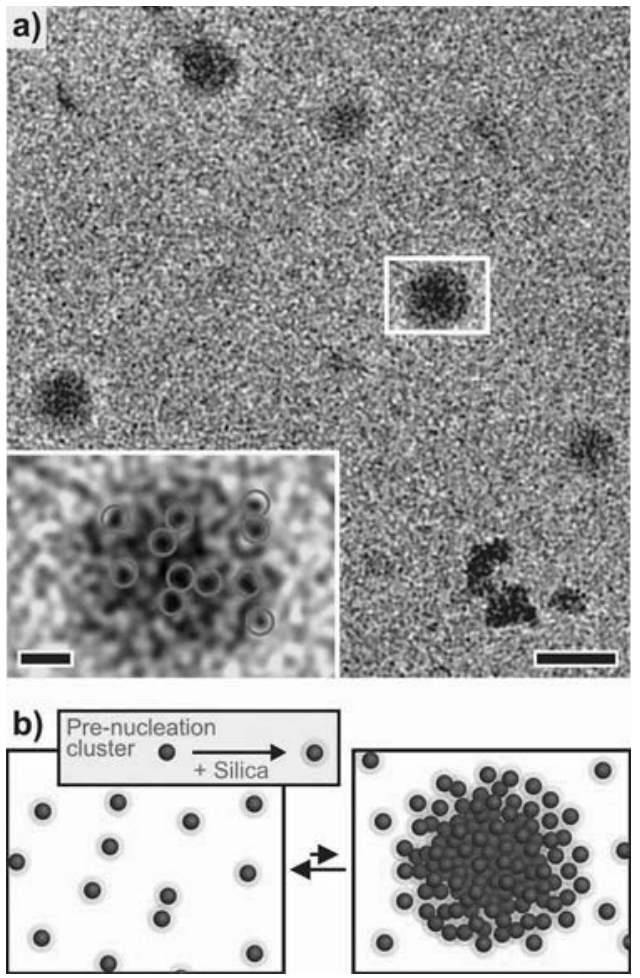

Figure 2. Dynamic cluster aggregation. a) Lower-magnification cryo-TEM image of a sample vitrified after $3 \mathrm{~min}$, showing larger objects next to myriad single cluster species. Scale bar: $50 \mathrm{~nm}$. Inset: Close-up view of the marked area, suggesting that the dark domains are loose aggregates of clusters (individuals are highlighted by red circles). Scale bar: $10 \mathrm{~nm}$. b) Schematic drawing visualizing cluster agglomeration under the influence of silica at $\mathrm{pH}$ 11. Note that structures are not drawn to scale and that the blue halo in the scheme is meant to generally indicate the presence of silicate species in the periphery of prenucleation clusters, rather than necessarily representing a skin of silica around them.

presence of silica in their periphery under the given conditions. In another approach, cluster sizes were estimated on the basis of the sedimentation coefficient utilizing the density of ACC, as described elsewhere. ${ }^{[20]}$ Resulting diameters were 3.34 and 3.21 $\mathrm{nm}$, which is in good agreement with values obtained using the Stokes-Einstein equation (4.61 and $2.53 \mathrm{~nm}$ ).

\subsection{Cluster Aggregation}

Occasionally, cryo-TEM images of freshly prepared solutions show bigger diffuse entities with typical dimensions of 20-50 nm (Figure 2a), which seem to be aggregates of loosely agglomerated single nanoclusters (Figure $2 \mathrm{~b}$ ) and thus probably still represent the prenucleation stage. DLS experiments corroborate the presence of two particle populations-individual clusters with hydrodynamic diameters below $10 \mathrm{~nm}$, and distinct cluster aggregates with sizes ranging from 20 to $200 \mathrm{~nm}$ (Figure 3a). Species with diameters larger than $50 \mathrm{~nm}$ likely correspond to interconnected primary aggregates (Figure S2 in the Supporting Information).

Small-angle X-ray scattering (SAXS) measurements confirm the results from DLS and identify multiple states of aggregation 

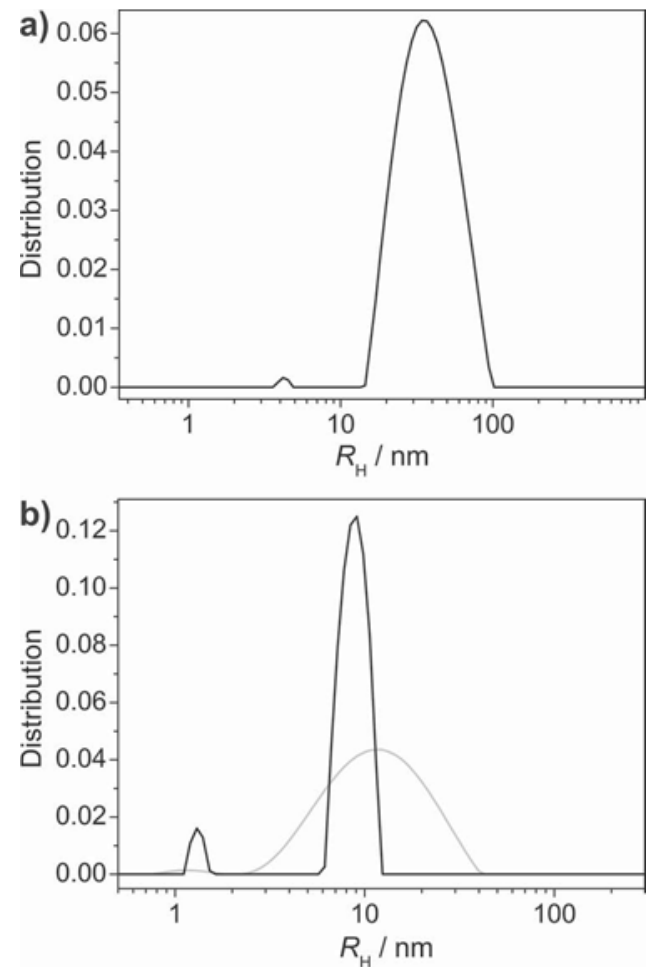

Figure 3. a) Size distribution diagram calculated on the basis of DLS measurements initiated 2 min after mixing with an acquisition time of $10 \mathrm{~min}\left(\left[\mathrm{Ca}^{2+}\right]=\left[\mathrm{CO}_{3}{ }^{2-}\right]=5 \mathrm{~mm}, 3720 \mathrm{ppm}\right.$ silica, $\left.\mathrm{pH} 11\right)$. The dominant peak covering radii between 10 and $100 \mathrm{~nm}$ belongs to cluster aggregates (Figure 2a), while the weak feature at $\approx 4 \mathrm{~nm}$ is assigned to individual clusters (Figure 1a,b). Note that sizes determined for single clusters by DLS exceed those measured in the cryo-TEM images (Figure 1c), presumably due to the presence of hydration layers around the clusters and errors in size estimations based on electron microscopy images. b) Size distributions of a sample that was centrifuged for $90 \mathrm{~min}$. The black curve corresponds to the state of the solution straight after centrifugation, while the grey curve has been recorded after subsequent equilibration for 1 day (measurement duration in both cases: $10 \mathrm{~min}$ ). Note that cluster diameters measured directly after centrifugation $(2.5-3.5 \mathrm{~nm})$ agree well with mean sizes detected by SAXS ( $3 \mathrm{~nm}$, Figure 4) as well as AUC data (2-5 nm; Table S1), thus most likely representing the true dimensions of the clusters.

(Figure 4), in agreement with the cryo-TEM data. Moreover, the mean size indicated for single clusters by SAXS is about $3 \mathrm{~nm}$ and thus complies well with values obtained by AUC, but on the other hand significantly exceeds those estimated on the basis of cryo-TEM images (Figure 1c). These discrepancies probably arise from shortcomings associated with the interpretation of electron contrast for such small species, ${ }^{[22]}$ and the fact that the scattering techniques (as well as AUC) detect hydration layers around the clusters, which may be enhanced by the presence of charged silicate oligomers.

Continuous DLS monitoring demonstrates that, subsequent to an induction period of several hours, the average size of the aggregates remains approximately constant $\left(R_{\mathrm{H}, \mathrm{av}} \approx 35 \mathrm{~nm}\right)$ with time (Figure 5). The overall scattering intensity, which is a direct measure for the actual concentration of the larger population, first increases and then gradually levels off after around $5 \mathrm{~h}$. This implies that aggregation proceeds steadily over hours.

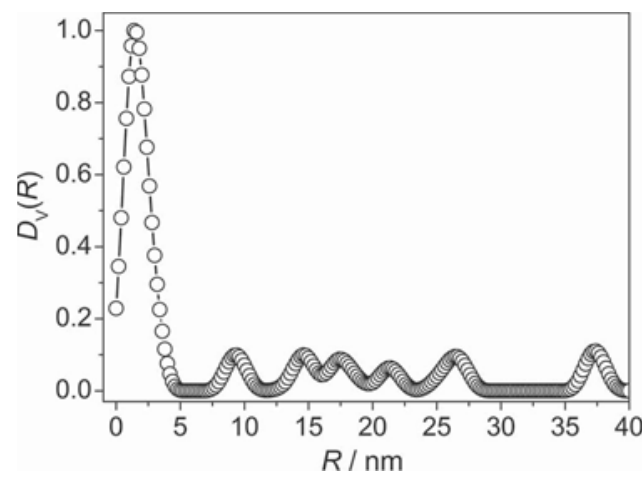

Figure 4. Volume-weighted radius distribution function $D_{\mathrm{V}}(R)$ derived on the basis of SAXS data collected directly from as-prepared solutions after an equilibration period of $30 \mathrm{~min}\left(\left[\mathrm{Ca}^{2+}\right]=\left[\mathrm{CO}_{3}{ }^{2-}\right]=5 \mathrm{~mm}, 3720 \mathrm{ppm}\right.$ silica, $\mathrm{pH} 11)$. The strong peak at $R<5 \mathrm{~nm}$ suggests that the majority of clusters exists in an isolated state in the solution, while several smaller features at radii between 10 and $40 \mathrm{~nm}$ indicate cluster assemblies with different aggregation numbers.

The rather narrow size distribution of the primary aggregates may be related to the generation of charge that restricts further aggregation beyond a critical size. ${ }^{[30]}$ Cryo-TEM analyses of equilibrated samples verify that although aggregated structures occur more frequently, the number of single clusters is still overwhelming (Supporting Information, Figure S2). Afterwards, no further changes could be discerned with neither of the used methods, even when the system was aged for more than a year. Therefore, we conclude that single nanoclusters and cluster aggregates co-exist permanently once the underlying aggregation processes have established a state of dynamic equilibrium (as depicted by Figure $2 \mathrm{~b}$ ). The fact that the size distributions found upon ageing are more or less constant moreover suggests that a competition between the rates of ion incorporation and release in prenucleation clusters determines the limited size of the different species, ${ }^{[24]}$ hence confirming their prenucleation stage character.

Additional DLS experiments have evidenced that the rate and final degree of cluster aggregation can be correlated with the speciation of silica in solution (data not shown). For example,

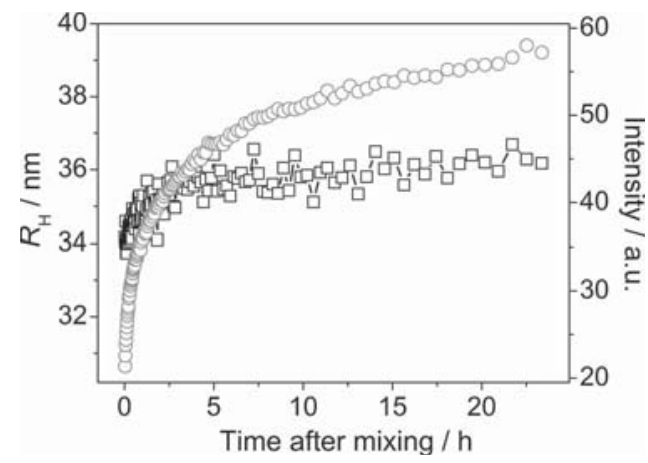

Figure 5. Continuous in situ DLS monitoring. Apparent hydrodynamic radii $R_{H}$ (black squares) obtained from monomodal fits to experimental autocorrelation functions collected at distinct times after mixing, and the evolution of the scattering intensity (grey circles) as a function of time $\left(\left[\mathrm{Ca}^{2+}\right]=\left[\mathrm{CO}_{3}{ }^{2-}\right]=5 \mathrm{~mm}, 3720 \mathrm{ppm}\right.$ silica, $\left.\mathrm{pH} 11\right)$. 
using an aged silica sol resulted in an increase in both the final average agglomerate size and the rate of aggregation. Raising stepwise the amount of added carbonate at a constant $\mathrm{Ca}^{2+}$ concentration also led to an increase in the hydrodynamic radius of the cluster agglomerates, while gradual aggregation to eventually larger entities was observed when the concentrations of all species were doubled. These observations corroborate that the periphery of the clusters is rich in silica and that cross-linking of the clusters might be assisted by inter-particle silica polymerization. Presumably, the silica interacts with the clusters by replacing water and/or hydroxide ions ${ }^{[13]}$ in the hydration shell around them. In solutions at $\mathrm{pH} 11$, the density of negative charges on siliceous species is high, ${ }^{[31]}$ rendering the net forces between silica-bearing clusters predominantly repulsive. Cluster aggregation is unfavorable under these circumstances, and only few agglomerates exist in equilibrium with a preponderant fraction of separate clusters (Figure 2b). Screening by the silica impedes direct cluster-cluster contact in the aggregates, such that nucleation via aggregation (i.e., pathway (ii)) remains inhibited in the long term.

Interestingly, larger cluster aggregates can be removed by centrifugation (16060 g, $90 \mathrm{~min}$ ). Remaining species showed mean hydrodynamic radii of roughly $10 \mathrm{~nm}$, while the overall scattering intensity was markedly decreased. Size distribution functions acquired immediately after completed centrifugation indicate an increased fraction of isolated clusters with $R_{\mathrm{H}} \approx$ $1.3 \mathrm{~nm}$ next to aggregates of around $9 \mathrm{~nm}$ in radius (Figure 3b). Initially narrow, the signals broaden when the sample is aged for a day, while no further changes were observed at later times. This confirms that the different aggregated states are in dynamic equilibrium as outlined above, since on average smaller agglomerates are formed after centrifugation owing to the lower overall $\mathrm{CaCO}_{3}$ content. The diameter obtained by DLS for individual clusters after preceding centrifugation $(2.6 \mathrm{~nm})$ nearly coincides with sizes determined independently by SAXS and AUC measurements $(\approx 3 \mathrm{~nm})$. These values are thus experimentally reproducible and considered realistic, as opposed to size data derived from cryo-TEM images only.

\subsection{Variation of $\mathrm{pH}$}

The capability of silica to stabilize the system against nucleation essentially depends on the $\mathrm{pH}$ of the medium, which is known to be a crucial parameter for the speciation of silica in solution. ${ }^{[29,31]}$ Decreasing the $\mathrm{pH}$ leads to enhanced protonation of siliceous species, and should therefore result in diminished mutual repulsion of clusters that are colloidally stabilized by silica. This notion is clearly evidenced by cryo-TEM analyses of samples with different initial $\mathrm{pH}$ values (Figure 6). At $\mathrm{pH}$ 10.3, the situation encountered $1 \mathrm{~min}$ after mixing resembles that at $\mathrm{pH} 11$, with individual clusters abounding and co-existing with a certain fraction of rather small aggregates (Figure 6a). After $1 \mathrm{~h}$, a considerable amount of larger agglomerates has developed $(\approx 20-40 \mathrm{~nm}$, Figure $6 \mathrm{~b})$; with time, these spheroidal entities interconnect progressively to build spacious network structures (Figure 6c). Extensive cross-linking is likely governed by silica condensation processes, which are accelerated at lower $\mathrm{pH}^{[31]}$ and may also involve the silica associated to the clusters.
Consequently, a siliceous matrix inclosing $\mathrm{CaCO}_{3}$ prenucleation clusters is generated and, owing to on-going polycondensation, eventually segregates from the solution to form a hydrogel. A closer look at the texture of the more compact objects in the network reveals that they are constituted of smaller primary units $(2-3 \mathrm{~nm})$, embedded in a lighter framework (Figure 6b). Apparently, aggregation of prenucleation clusters at this $\mathrm{pH}$ level frequently leads to assemblies which are denser than at higher $\mathrm{pH}$. However, individual clusters inside these agglomerates do not merge to a noticeable extent and we could neither trace significant changes in terms of local structure on further ageing (Figure $6 \mathrm{c}$ ), nor does crystallization occur. This implies that although cluster aggregation is much more pronounced in the system, the silica still protects individuals from fusion and inhibits nucleation (Figure 6d, upper pathway).

When the $\mathrm{pH}$ is decreased to values below 10, colloidal stabilization is in turn completely suspended (Figure 6d, lower pathway). At $\mathrm{pH}$ 9.3, the sample taken after 1 min already contains loosely aggregated networks in some instances (Figure 6e). After $30 \mathrm{~min}$, inhomogeneous conglomerates spanning across micron-scale dimensions can be discerned (Figure $6 f$ ). The darker particles within these networks are somewhat larger (up to $10 \mathrm{~nm}$ ) than those found at higher $\mathrm{pH}$, indicating fractional cluster coalescence. As time proceeds, a novel population of fairly consolidated spherical particles with diameters of around $30 \mathrm{~nm}$ is occasionally identified next to the networks at some sites (Figure $6 \mathrm{~g}$ and Supporting Information, Figure S3). These particles are prone to aggregation and electron diffraction (ED) patterns suggest the presence of partially crystalline material (inset in Figure 6g); however, we also observed purely amorphous nanoparticles (Supporting Information, Figure S4). These findings evidence that primary ACC grains form via aggregation of prenucleation clusters, as observed in previous studies on $\mathrm{CaCO}_{3}$ crystallization in the absence of additives. ${ }^{[20,23]}$ Upon continued ripening, the metastable ACC particles eventually transform to yield numerous micron-sized calcite crystals (Figure 6h and Supporting Information, Figure S5). Essentially, the behavior traced at $\mathrm{pH} 9.3$ shows that stabilization at higher $\mathrm{pH}$ values is not based on incorporation of silica into the calcium carbonate precursors, but rather relies on screening of individual clusters, which becomes less efficient with decreasing $\mathrm{pH}$. If silica was incorporated in the clusters, the reduced charge and the increased propensity of silica to undergo condensation at lower $\mathrm{pH}$ should still (and likely even more effectively) inhibit nucleation.

The notion that insufficient protection against nucleation below $\mathrm{pH} 10$ originates from a reduced number of charges on the clusters and lacking mutual repulsion is corroborated by Zeta potential measurements. In general, results obtained for the different samples (Supporting Information, Table S3) are in good agreement with values reported in literature for silica surfaces in contact with solutions at corresponding $\mathrm{pH}$ levels and comparable ionic strengths $(\mathrm{I} \approx 80 \mathrm{~mm}$ in the present experiments) ${ }^{[32]}$ This appears reasonable when assuming that the $\mathrm{CaCO}_{3}$ clusters are covered by silicate species. In particular, measurements for solutions at $\mathrm{pH} 11.0$ and 10.3 gave Zeta potentials which are equal within experimental error (ca. $-35 \mathrm{mV}$ ), whereas the value determined for $\mathrm{pH} 9.3$ is significantly lower (ca. $-20 \mathrm{mV}$ ). Calculations of potential energy profiles based 


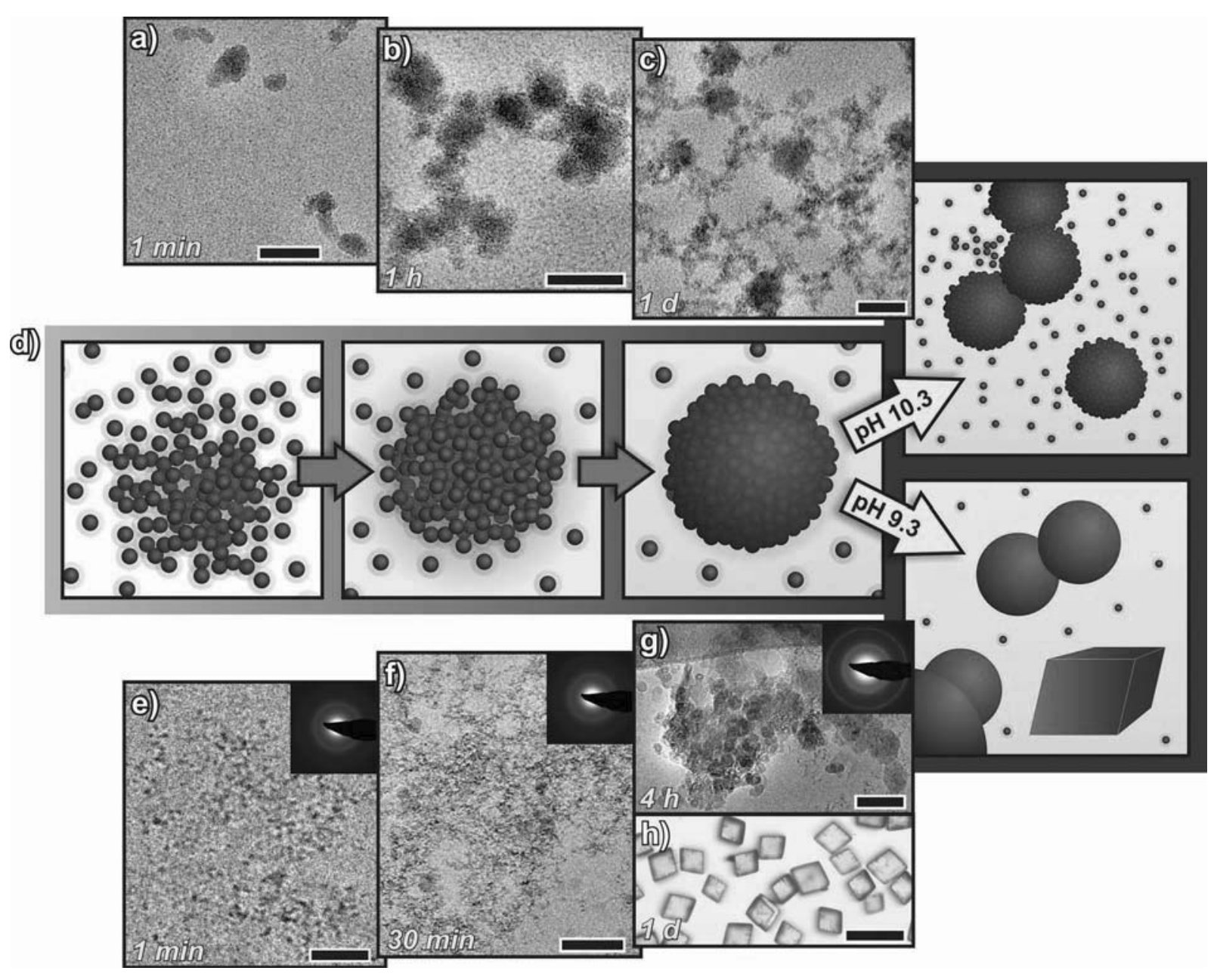

Figure 6. Effect of $\mathrm{pH}$ on the degree of silica-mediated inhibition of $\mathrm{CaCO}_{3}$ nucleation. a-c) Cryo-TEM images taken at different times after mixing from samples with an initial pH of 10.3. Scale bars: $50 \mathrm{~nm}$. d) Schematic sequence illustrating the aggregation behavior of silica-decorated prenucleation clusters at lower $\mathrm{pH}$. Cluster coalescence inside the aggregates and nucleation of ACC nanoparticles only occur below a certain threshold in $\mathrm{pH}$. e-h) Microscopy images representing the temporal evolution of the system at a starting $\mathrm{pH}$ of 9.3. ED patterns (shown as insets in (e-g)) indicate that the open cluster networks formed within $30 \mathrm{~min}$ are still amorphous. After $4 \mathrm{~h}$, agglomerates of more compact particles are observed in which some crystallinity is already inherent. Equilibration for about 1 day results in a multitude of rhombohedral crystals ( $h$ ), which were confirmed to be calcite by X-ray diffraction (Figure S5 in the Supporting Information). Scale bars: e) $50 \mathrm{~nm}, \mathrm{f}, \mathrm{g}) 100 \mathrm{~nm}$, and h) $100 \mu \mathrm{m}$.

on DLVO theory indicate that a distinct barrier must be overcome in order to allow colloids to coalesce in samples at $\mathrm{pH}$ 11.0 and $10.3(\approx 1.1 \mathrm{kT})$; in turn, this is not the case at $\mathrm{pH} 9.3$ $(\approx 0.3 \mathrm{kT}$; see Supporting Information, Figure S6). This suggests poor colloidal stability for the system at low $\mathrm{pH}$, which is in line with the cryo-TEM data. We note that the estimated energy barriers are relatively small, since colloidal dispersions are usually considered to be stabilized against flocculation for a barrier height of $\geq 10 \mathrm{kT} .{ }^{[33]}$ This may imply that steric effects contribute to stabilization in the present case, as proposed in previous work to explain the anomalous coagulation behavior of silica sols. ${ }^{[34]}$ However, parameter variation showed that the by far largest error in our calculations is related to the use of the Hamaker constant of amorphous silica. It applies for a bulk phase and will therefore strongly overestimate the attractive potential between hydrated silica oligomers bound in the periphery of the clusters. As a consequence, the true magnitude of the attractive potential should be lower than estimated, thus increasing the energy barrier in accordance with recent reviews on DLVO theory. ${ }^{[35]}$ Taken together, the Zeta potential data support lacking electrostatic repulsion at $\mathrm{pH} 9.3$ and hence verify the notion that silica-decorated clusters can aggregate under these conditions to a much larger extent than at higher $\mathrm{pH}$. In addition to this "classical" mechanism of colloidal destabilization, deficient inhibition of nucleation at low $\mathrm{pH}$ could be promoted by enhanced silica condensation rates expected in this case, ${ }^{[31]}$ as fast polymerization might prevent tight integration of the clusters into the forming siliceous network and/or withdraw oligomeric silicate species from their periphery.

\subsection{Cluster Isolation}

Remarkably, the stabilizing effect of silica at high $\mathrm{pH}$ is strong enough to enable straightforward isolation of the clusters in large amounts. To that end, solutions containing clusters and 


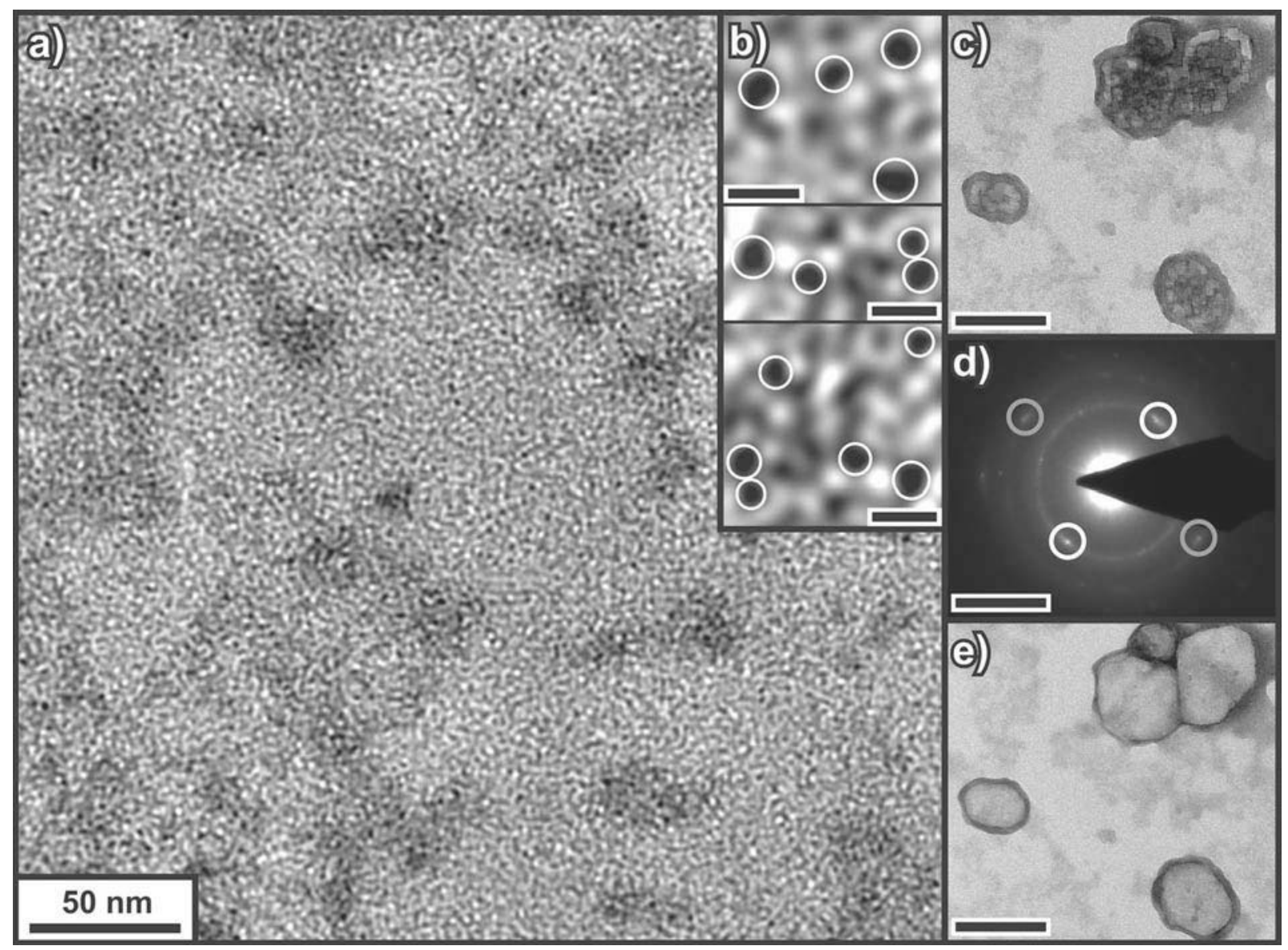

Figure 7. Silica-stabilized $\mathrm{CaCO}_{3}$ nanoclusters in dry state. a) TEM image of the residue obtained upon drying aliquots of dialyzed solution, showing myriads of isolated tiny species dispersed over the whole image area. The determined average diameter of $1.2 \pm 0.8 \mathrm{~nm}$ (measuring 200 individuals) nearly coincides with the mean value obtained for the clusters observed in cryo-TEM images $(1.1 \pm 0.6 \mathrm{~nm})$. b) Zooms into selected regions of (a) revealing single cluster species (white circles) with dimensions in the range of 1-2 nm (scale bars: $5 \mathrm{~nm}$ ). c) Nanocrystals found at a different site on the grid, likely formed due to insufficient protection of a certain fraction of clusters against nucleation during dialysis and/or drying. Fragmentation of the crystals indicates ongoing decomposition (scale bar: $100 \mathrm{~nm}$ ). d) Electron diffraction pattern of the crystals, displaying faint spots along two discrete rings (scale bar: $4 \mathrm{~nm}^{-1}$ ). Measured distances in-between pairs of reflections correspond to lattice spacings of $3.02 \AA$ (white circles) and $2.16 \AA$ (grey circles). With respect to the common limits of error in electron diffraction, reflections can be indexed to the (104) and (202) planes of calcite (database spacings: C-104: $3.035 \AA$ A C-202: $2.095 \AA$ ). e) Crystals after exposure to the electron beam during diffraction analysis. Decomposition of the beam-sensitive carbonate material left hollow particles with margins rich in Ca (verified by EDX elemental mapping) (scale bar: $100 \mathrm{~nm}$ ).

cluster aggregates were dialyzed to remove excess salt and subsequently dried by lyophilization. Alternatively, cluster separation was achieved by direct freeze-drying, induced precipitation of a silica framework containing embedded clusters, or via preparative ultracentrifugation, as discussed in the Supporting Information (Section S4). TEM images of the residue left upon drying dialyzed solution on a grid are characterized by a multitude of discrete and predominantly separated nanoclusters (Figure 7). This indicates that the structural integrity of the clusters was not affected during the applied procedure. While the size and number of individual clusters is comparable to what was found by cryo-TEM images for the solution state (Figure 1), the overall frequency of cluster aggregates is lower, suggesting that some of them dissociated during dialysis-as expected if they form on basis of equilibrium thermodynamics. Next to the clusters, larger particles with typical sizes of 50-100 nm were observed (Figure 7c) Diffraction patterns acquired from these objects show crystalline reflections that can be assigned to the calcite lattice (Figure 7d). Prolonged exposure to the electron beam $(\approx 3 \mathrm{~s})$ led to continuous disintegration of the crystals (presumably due to thermal decarboxylation of $\mathrm{CaCO}_{3}$ in high vacuum), ${ }^{\left[{ }^{[6]}\right.}$ so that elemental analyses of intact particles were not possible. Their remnants, however, proved to be rich in calcium. The occurrence of calcite crystals in the images demonstrates that shielding of the clusters is not perfectly efficient under the conditions during sample preparation, likely due to the decrease in the silica concentration in the course of dialysis. Since IR and XRD results for samples obtained by direct freezedrying do not hint at the presence of calcite or other crystalline phases (see below), partial crystallization must be due to the slow evaporation process on the TEM grid.

Probing the chemical nature of the clusters by means of EDX spectroscopy and elemental mapping failed because of their beam sensitivity. Marked degradation was discernible already 

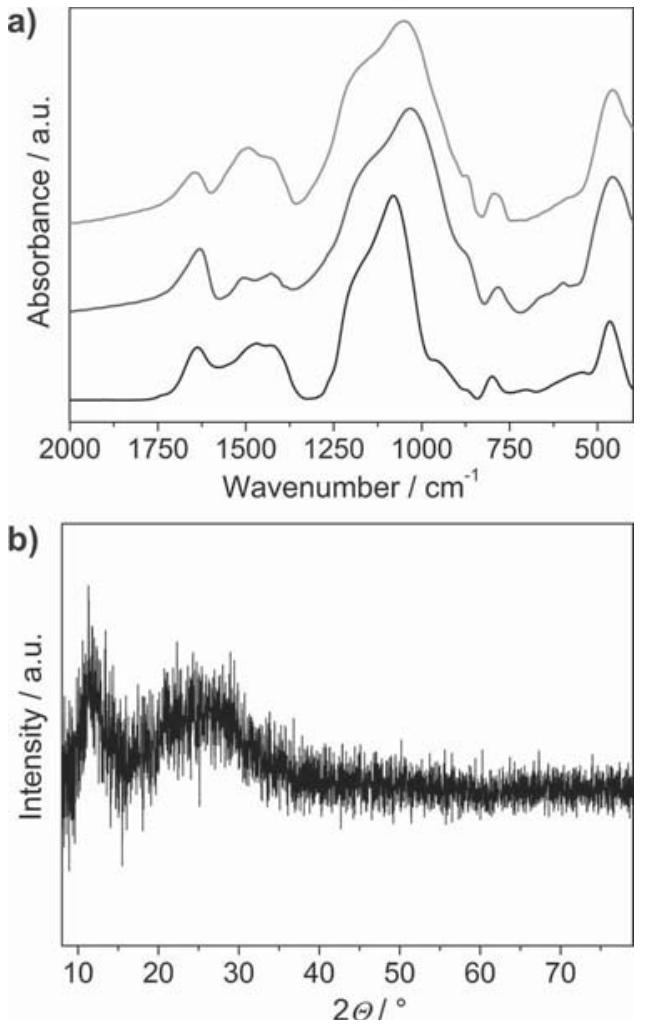

Figure 8. a) Vibrational spectra of powders obtained by lyophilization of dialyzed solutions (lower curve), ultracentrifugation and subsequent freeze-drying of the gel body deposited on the bottom of the vial (middle curve), and filtration of the precipitate formed upon adding $\mathrm{NaCl}$ (upper curve) (see Section S3 in the Supporting Information for experimental details). The initial compositions of the solutions were $\left[\mathrm{Ca}^{2+}\right]=\left[\mathrm{CO}_{3}{ }^{2-}\right]$ $=5 \mathrm{~mm}, 3720 \mathrm{ppm}$ silica for both dialysis and ultracentrifugation, and $\left[\mathrm{Ca}^{2+}\right]=\left[\mathrm{CO}_{3}{ }^{2-}\right]=5 \mathrm{~mm},[\mathrm{NaCl}]=0.2 \mathrm{M}, 3720 \mathrm{ppm}$ silica in case of induced precipitation. b) Background-subtracted WAXS diffractogram of the gel sediment formed upon ultracentrifugation, verifying that the sample is entirely amorphous and thus corroborating that the powder contains ACC.

after acquisition of a single image, and the clusters had quantitatively vanished upon longer irradiation times. Ultimately, Ca intensities were evenly distributed and low in the elemental maps. In turn, when analyzing cluster aggregates, we found increased counts of Si even after decomposition of calcium carbonate was completed. This means that silica is stable under the given operating conditions (as confirmed also by studies of neat silica reference samples) and, hence, that the observed clusters do not consist of silica.

IR and XRD data demonstrate that the $\mathrm{CaCO}_{3}$ component in the isolated samples relates to an ACC-like phase (see Figure 8 and Section S4 in the Supporting Information). Infrared spectra recorded from powders obtained by dialysis, centrifugation and induced precipitation show strong absorption in the range of $900-1300 \mathrm{~cm}^{-1}$, which is typical for amorphous silica and can be attributed to Si-O-Si stretching lattice modes (Figure 8a). ${ }^{[37]}$ Further bands at around 450, 800, and $1630 \mathrm{~cm}^{-1}$ originate from bond-rocking and bond-bending vibrations of silica, and deformation modes of entrapped water molecules, respectively (see Table S4 in the Supporting Information for a detailed assignment of the different peaks). Prominent bands of $\mathrm{CaCO}_{3}$ are generally located at $690-750 \mathrm{~cm}^{-1}\left(v_{4}\right.$-mode), $850-880 \mathrm{~cm}^{-1}$ ( $v_{2}$-mode) and $1420-1490 \mathrm{~cm}^{-1}$ ( $v_{3}$-mode), depending on polymorphism. ${ }^{[38]}$ As opposed to crystalline phases, ACC can be distinguished by a splitting of the $v_{3}$-peak and a marked broadening or disappearance of the $v_{4}$-absorption. ${ }^{[39]}$ These two criteria are clearly fulfilled by the spectra displayed in Figure 8a, indicating that the major carbonate phase present is ACC. This is supported by the observed wavenumber of the $v_{2}$-band, which approximates values reported for ACC in literature $\left(866 \mathrm{~cm}^{-1}\right) .{ }^{[39]}$ Thus, all of the applied procedures yielded powders containing a considerable amount of $\mathrm{CaCO}_{3}$ clusters embedded in a matrix of amorphous silica. We note that differences in the absorption maxima of the siliceous component may hint at varying degrees of condensation; for example, the bond-stretching vibration in the dialyzed sample is shifted to comparably high wavenumbers, suggesting a highly crosslinked structure. In this case, a distinct band at about $950 \mathrm{~cm}^{-1}$ can be discerned that may be due to stretching vibrations of free silanol $(\mathrm{Si}-\mathrm{OH})$ groups. $^{[37]} \mathrm{X}$-ray diffraction analyses confirm that all isolated powders are amorphous and hence corroborate the notion of ACC-like clusters. As shown exemplarily for the sample prepared by centrifugation in Figure $8 \mathrm{~b}$, the acquired patterns indicate the presence of amorphous structures with some ordering, in agreement with previous studies on amorphous silica ${ }^{[40]}$ and ACC. ${ }^{[41]}$

\subsection{Clusters in Reference Samples}

As already mentioned above, AUC measurements of reference samples lacking either calcium or carbonate also suggested the presence of cluster-like species, the origin of which remains to be discussed. First of all, we emphasize that neat silica sols did not show noticeable correlation in light scattering experiments (data not shown), thus ensuring that the existence of nanoparticles is directly related to the presence of $\mathrm{CaCl}_{2}$ and $\mathrm{Na}_{2} \mathrm{CO}_{3}$, respectively. Indeed, DLS data support the occurrence of clusters with radii of 2-7 $\mathrm{nm}$ in the absence of $\mathrm{Ca}^{2+}$, next to a larger fraction with $R_{\mathrm{H}, \mathrm{av}} \approx 50 \mathrm{~nm}$ (Supporting Information, Figure S7). Corresponding cryo-TEM images show that cluster-like species are quite rare in this system (Supporting Information, Figure S8). Nevertheless, they can be detected by DLS because the amount of larger particles is also rather small. In typical images of samples without added carbonate, polydisperse spherical particles with diameters ranging from 20 to $40 \mathrm{~nm}$ are observed, which occasionally become subject to agglomeration (Figure S8). In addition, the solutions contain a considerable number of nanoclusters (as judged by cryo-TEM) which, however, are not resolved in DLS (Supporting Information, Figure S8). This indicates that the concentration of clusters relative to larger particles is lower in this system than in a mixture of silica and both $\mathrm{CaCl}_{2}$ and $\mathrm{Na}_{2} \mathrm{CO}_{3}$ (where individual clusters were detected next to cluster aggregates; Figure 2).

The formation of cluster-like species in the absence of carbonate ions may be due to $\mathrm{Ca}^{2+}$-induced silica polymerization, owing to the strong "salting-out" effect of hard divalent cations, ${ }^{[42]}$ which triggers silica condensation by screening otherwise repulsive charges. ${ }^{[31]}$ Moreover, small polymeric particles 
with sizes of only few nanometers and spherical colloids measuring several tens of nanometers are common in the course of silica precipitation from solution. ${ }^{[43]}$ These considerations raise the question whether the clusters found in the actual samples could also consist of silica or calcium silicates, rather than directly involving calcium carbonate. A crucial issue to clarify in this context is whether the calcium ions merely assist in the polymerization process or if they are co-precipitated with silica. In the latter case, $\mathrm{Ca}^{2+}$ would not be available for $\mathrm{CaCO}_{3}$ formation, whereas sheer colloidal interactions should allow for $\mathrm{CaCO}_{3}$ crystallization, especially with regard to the high supersaturation of the system. To elucidate this, experiments were conducted in which silica sols were coagulated by adding $\mathrm{CaCl}_{2}$. Analyses of the flocculated material showed that it essentially consists of amorphous silica (no calcium silicate), with minor amounts of calcium entrapped between the coagulated particles (data not shown). Precipitation of calcium-rich silica particles in the samples can therefore be ruled out and, consequently, we expect the major fraction of the $\mathrm{Ca}^{2+}$ ions to be free and available for interaction with carbonate under the experimental conditions.

Enhanced silica condensation can in turn hardly account for nanoparticle formation in mixtures of $\mathrm{Na}_{2} \mathrm{CO}_{3}$ and silica. Here, we speculate that the observed nanoscale species are clusters of sodium carbonate. In fact, clustering of well-soluble salts has been demonstrated in supersaturated solutions. ${ }^{[4]}$ Coating by silica may cause a shift in the equilibrium with the free ions in favor of the bound state, thus increasing the number of clusters to a measurable amount also in dilute systems. Indeed, the existence of $\mathrm{Na}_{2} \mathrm{CO}_{3}$ clusters in silica-containing solutions is supported by IR and XRD data recorded from powders obtained by direct freeze-drying (see Section S3 in the Supporting Information), and their rare occurrence as compared to $\mathrm{CaCO}_{3}$ would qualitatively correlate with the solubility of the two salts.

With respect to the above, we cannot exclude that polymeric silica particles and silica-associated clusters of other salts-both with similar sizes-co-exist with clusters of calcium carbonate in the samples. However, the temporal evolution of the system traced at different initial $\mathrm{pH}$ values (Section 2.3) and, importantly, the analyses performed on isolated clusters (Section 2.4) provide clear evidence that the vast majority of cluster species observed in the solutions are indeed composed of $\mathrm{CaCO}_{3}$.

\section{Conclusions}

Our results show that in the presence of silica and at sufficiently low $\mathrm{pH}$, nucleation of calcium carbonate can proceed in the same manner as it has been reported in the absence of additives, ${ }^{[20,23]}$ that is, via aggregation of prenucleation clusters. Phase separation thereby appears to rest upon the coalescence and fusion of clusters within transient aggregates, which might be triggered by the release of water from the inside of the aggregates driven by osmotic pressures, and could rely on structural changes of the clusters. ${ }^{[22,24]}$ Continued cluster coalescence eventually leads to the precipitation of ACC nanoparticles, which subsequently crystallize. The role of silica in the present experiments is to decelerate the processes that underlie nucleation via colloidal stabilization of prenucleation clusters against aggregation. The collected data clearly evidence that the degree of this stabilization correlates with the chemical speciation of silica, which is $\mathrm{pH}$-dependent. While aggregation of silica-decorated prenucleation clusters can proceed gradually at lower $\mathrm{pH}$ values (where silica species are largely protonated and prone to condensation), this is no longer possible at elevated $\mathrm{pH}$ due to the high number of repulsive charges at silica-bearing clusters. Under these conditions, nucleation of calcium carbonate remains prevented in the long term. The detailed mode of silica binding on the clusters-which is the basis for colloidal stabilization-remains uncertain. Possible scenarios include a mechanism involving local $\mathrm{pH}$ gradients around growing carbonate clusters as described for ACC particles, ${ }^{[28,45]}$ as well as electrostatic interactions between oligomeric silicate species and the $\mathrm{Ca}^{2+}$ ions in the periphery of the clusters assisted by water molecules, in analogy to the effect of polyacrylate. ${ }^{[46]}$ The latter may be driven by a gain in entropy ${ }^{[4]}$ and would explain the poor stabilization found at lower $\mathrm{pH}$.

As silica appears to interact only superficially with the clusters (i.e., it is likely not incorporated), the observed longterm stabilization against nucleation at high $\mathrm{pH}$ indicates that internal structural rearrangements within prenucleation clusters (pathway (i)) are improbable, if not impossible, under the given conditions. This might be due to an enormous activation barrier which inhibits nucleation for more than a year. However, in our opinion, such a large extent of kinetic stabilization is unlikely and it seems more plausible that thermodynamic aspects play an important role. In other words, our findings tentatively support a crossover in stability at the nanoscale, with ACC-like structural forms becoming more stable than crystalline ones, in line with predictions of computer simulations. ${ }^{[27]}$ One may even speculate that, at the length scale typical for prenucleation clusters, their chain-like structural form ${ }^{[24]}$ is the stable conformation of calcium carbonate. Although our data do certainly not allow claiming that pathway (i) is blocked in general, there are indications that it might indeed be improbable also in the absence of silica, that is, under common homogeneous conditions.

Regardless of whether nucleation is governed by kinetic and/ or thermodynamic factors in the presence of silica, the experiments performed in this work demonstrate that colloidal stabilization by silicate oligomers enables isolation of $\mathrm{CaCO}_{3}$ prenucleation clusters in large amounts, while preventing precipitation of a bulk phase. This is a highly valuable observation, as the developed isolation protocols may be utilized for the preparation of samples rich in prenucleation clusters, thus facilitating future in-depth investigations of these elusive precursors. It remains to be proven, though, if the structural integrity of the solute clusters is indeed preserved upon transfer into dry state.

In addition, our results provide new insight into the nature of prenucleation clusters as such, and a comparison of the present data with previous studies leads to three important conclusions. First, the size distributions determined by light and X-ray scattering are rather broad and their shapes conform to the theoretical speciation of highly dynamic polymers of ion pairs. ${ }^{[24,48]}$ Second, the mean (hydrodynamic) diameter found for the clusters is about $3 \mathrm{~nm}$ which, for a random coil $\left(R_{\mathrm{h}} / R_{\mathrm{g}} \approx 0.66\right),{ }^{[49]}$ corresponds to a gyration radius of $\approx 2.3 \mathrm{~nm}$. This compares 
reasonably well to results of simulations, which estimate the radius of gyration for a cluster comprising $36 \mathrm{CaCO}_{3}$ units to $0.9-1.2 \mathrm{~nm} .{ }^{[24]}$ Considering the uncertainty of this estimation and the notion that the clusters investigated in the present study are decorated by silica, it seems evident that prenucleation clusters of calcium carbonate contain tens of ion pairs on average, as already suggested in previous work. ${ }^{[20]}$ Third, AUC as well as DLS and SAXS analyses consistently yielded average sizes of around $3 \mathrm{~nm}$ for silica-bearing clusters, whereas measurements in electron microscopy images gave average values of ca. $1 \mathrm{~nm}$. Even though the diameters determined by scattering techniques and ultracentrifugation include the hydration shell of the clusters, this discrepancy indicates that cryo-TEM significantly underestimates the true dimension of these small species. This is likely due to insufficient electron contrast of large parts of the clusters, ${ }^{[22]}$ and must be taken into account when interpreting corresponding data.

Finally, we note that colloidal stabilization of solutions containing prenucleation clusters is an effective means to deliberately prevent crystallization and hence depicts a promising approach towards novel strategies for scale inhibition. Moreover, given that cluster-like species were reported also for a series of other minerals, ${ }^{[42,43,50-54]}$ we envisage that the concepts developed in this work and in particular the described isolation protocols may be applied to clusters of different materials as well.

\section{Experimental Section}

To provoke $\mathrm{CaCO}_{3}$ formation, aqueous solutions of calcium chloride and sodium carbonate (5 mL each, $2.5-20 \mathrm{~mm}$, usually equimolar) were combined rapidly with the aid of a graded pipette. Addition of silica was realized by dissolving $\mathrm{Na}_{2} \mathrm{CO}_{3}$ in dilutions of commercial sodium silicate solution ("water glass", diluted at ratios between 1-10 and 1-100 $(\mathrm{v} / \mathrm{v}))$, which are known to contain a complex mixture of different monomeric and oligomeric silicate species. ${ }^{[29]}$ The final molar $\mathrm{Si} / \mathrm{Ca}$ ratio in the samples was about 12.5 and hence substantially higher than silica concentrations used to stabilize ACC in a previous study. ${ }^{[28]} \mathrm{A}$ typical sample contained $5 \mathrm{~mm}$ each of $\mathrm{CaCl}_{2}$ and $\mathrm{Na}_{2} \mathrm{CO}_{3}, 62 \mathrm{~mm} \mathrm{SiO}_{2}$ (3720 ppm), and additional $48 \mathrm{mM} \mathrm{Na}^{+}$as counter-ions. The initial pH was $11.00 \pm 0.05$ and, where indicated, set to lower values by adding $\mathrm{HCl}$ to the $\mathrm{CaCl}_{2}$ solution used for mixing.

For cryo-TEM, aliquots were drawn from the samples at different times and spread on lacey carbon grids by blotting with a filter paper. The resulting thin film was vitrified by quickly plunging the grids into liquid ethane at its freezing point. Specimens were examined at temperatures around $90 \mathrm{~K}$. Collected images were processed with a background-subtraction routine and, where appropriate, a smoothing filter was applied to reduce noise. For DLS, SAXS, Zeta potential, and AUC experiments, reagents were mixed accordingly and transferred to the respective sample cell after the desired period of ageing. Data acquisition was initiated immediately and measurements were performed continuously without any further intervention. Experimental details, procedures, and data evaluation routines are described in the Supporting Information (Section S3).

Isolation of the clusters was achieved by dialyzing as-prepared samples with membrane tubes retaining cluster species but letting pass ions and smaller silica oligomers, giving solutions rich in prenucleation clusters and freed from excess salt. Powders obtained upon drying were investigated by TEM, IR spectroscopy and X-ray diffraction. Alternative isolation methods and the characterization of corresponding products are summarized in the Supporting Information (Section S4).

\section{Acknowledgements}

The authors thank $H$. Santner (Anton Paar GmbH) for SAXS analyses, A. Völkel (MPI Golm) for performing the AUC experiments, M. Andratschke (University of Regensburg) for powder diffraction measurements, U. Aschauer (Princeton University) for providing the software Hamaker 2.1.0, J. Schmidt and E. Kesselman (Technion Haifa) for cryo-TEM imaging, and C.V.S.K. Chakravadhanula (University of Kiel) and R. Rachel (University of Regensburg) for TEM studies. Financial support by the Fonds der Chemischen Industrie (M.K.), BASF SE (M.K.), the program Juan de la Cierva of the Spanish Ministry of Science (MICINN) (E.M.G.), the Deutsche Forschungsgemeinschaft (SFB 481) (M.D.), and the MICINN projects "La Factoría de Cristalizacíon" and MAT2006-11701 (J.M.G.R.) is gratefully acknowledged.

[1] R. Becker, W. Döring, Ann. Phys. 1935, 24, 719.

[2] J. J. De Yoreo, P. Vekilov, Rev. Mineral. Geochem. 2003, 54, 57.

[3] H. Cölfen, M. Antonietti, Mesocrystals and non-classical crystallization, Wiley, Chichester, UK 2008.

[4] F. C. Meldrum, H. Cölfen, Chem. Rev. 2008, 108, 4332.

[5] L. Brečević, A. E. Nielsen, J. Cryst. Growth 1989, 98, 504.

[6] S. Weiner, I. Sagi, L. Addadi, Science 2005, 309, 1027.

[7] F. Nudelman, K. Pieterse, A. George, P. H. H. Bomans, H. Friedrich, L. J. Brylka, P. A. J. Hilbers, G. de With, N. A. J. M. Sommerdijk, Nat. Mater. 2010, 9, 1004.

[8] A. V. Radha, T. Z. Forbes, C. E. Killian, P. U. P. A. Gilbert, A. Navrotsky, Proc. Natl. Acad. Sci. USA 2010, 107, 16438.

[9] D. Gebauer, P. N. Gunawidjaja, J. Y. P. Ko, Z. Bacsik, B. Aziz, L. Liu, Y. Hu, L. Bergström, C. W. Tai, T. K. Sham, M. Eden, N. Hedin, Angew. Chem. Int. Ed. 2010, 49, 8889.

[10] J. Rieger, T. Frechen, G. Cox, W. Heckmann, C. Schmidt, J. Thieme, Faraday Discuss. 2007, 136, 265.

[11] L. B. Gower, Chem. Rev. 2008, 108, 4551.

[12] a) S. E. Wolf, J. Leiterer, M. Kappl, F. Emmerling, W. Tremel, J. Am. Chem. Soc. 2008, 130, 12342; b) S. E. Wolf, J. Leiterer, V. Pipich, R. Barrea, F. Emmerling, W. Tremel, J. Am. Chem. Soc. 2011, 133, 12642.

[13] S. E. Wolf, L. Müller, R. Barrea, C. J. Kampf, J. Leiterer, U. Panne, T. Hoffmann, F. Emmerling, W. Tremel, Nanoscale 2011, 3, 1158.

[14] B. Cantaert, Y. Y. Kim, H. Ludwig, F. Nudelman, N. A. J. M. Sommerdijk, F. C. Meldrum, Adv. Funct. Mater. 2012, 22, 907.

[15] H. A. Lowenstam, S. Weiner, On biomineralization, Oxford University Press, New York 1989.

[16] F. Lippmann, Sedimentary carbonate minerals, Springer, Berlin 1973.

[17] R. E. Zeebe, J. C. Zachos, K. Caldeira, T. Tyrell, Science 2008, 321, 51.

[18] W. Tegethoff, J. Rohleder, E. Kroker, Calcium carbonate: from the Cretaceous period to the 21st century, Birkhäuser, Basel, Switzerland 2001.

[19] J. Rieger, Tenside, Surfactants, Deterg. 2002, 39, 221.

[20] D. Gebauer, A. Völkel, H. Cölfen, Science 2008, 322, 1819.

[21] F. C. Meldrum, R. P. Sear, Science 2008, 322, 1802.

[22] D. Gebauer, H. Cölfen, Nano Today 2011, 6, 564. 
[23] E. M. Pouget, P. H. H. Bomans, J. A. C. M. Goos, P. M. Frederik, G. de With, N. A. J. M. Sommerdijk, Science 2009, 323, 1455.

[24] R. Demichelis, P. Raiteri, J. D. Gale, D. Quigley, D. Gebauer, Nat. Commun. 2011, 2, 590

[25] J. J. De Yoreo, unpublished.

[26] T. Z. Forbes, A. V. Radha, A. Navrotsky, Geochim. Cosmochim. Acto 2011, 75, 7893.

[27] P. Raiteri, J. D. Gale, J. Am. Chem. Soc. 2010, 132, 17623.

[28] M. Kellermeier, E. Melero-García, F. Glaab, R. Klein, M. Drechsler, R. Rachel, J. M. García-Ruiz, W. Kunz, J. Am. Chem. Soc. 2010, 132, 17859.

[29] S. Sjöberg, J. Non-Cryst. Solids 1996, 196, 51.

[30] G. H. Bogush, M. A.Tracy, C. F. Zukoski, J. Non-Cryst. Solids 1988 , 104, 95.

[31] R. K. Iler, The chemistry of silica, Wiley, New York 1979.

[32] P. J. Scales, F. Grieser, T. W. Healy, L. R. White, D. Y. C. Chan, Langmuir 1992, 8, 965.

[33] R. Buscall, R. H. Ottewil, in Polymer colloids (Eds: R. Buscall, T. Corner, J. F. Stageman), Elsevier, New York 1985, Ch. 5.

[34] T. W. Healy, in The colloid chemistry of silica, Vol. 234 (Ed: H. E. Bergna), American Chemical Society, Washington, DC 1994, Ch. 7.

[35] S. Croll, Prog. Org. Coat. 2002, 44, 131.

[36] A. Singh, S. Dash, M. Kamruddin, P. K. Ajikumar, A. K. Tyagi, V. S. Raghunathan, B. Raj, J. Am. Ceram. Soc. 2002, 85, 927.

[37] J. R. Martinez, F. Ruiz, Y. F. Vorobiev, F. Perez-Robles, J. GonzalezHernandez, J. Chem. Phys. 1998, 109, 7511.
[38] F. A. Andersen, L. Brečević, Acta Chem. Scand. 1991, 45, 1018.

[39] L. Addadi, S. Raz, S. Weiner, Adv. Mater. 2003, 15, 959.

[40] A. Sobczynski, Monatsh. Chem. 1992, 123, 211.

[41] a) M. Faatz, F. Gröhn, G. Wegner, Adv. Mater. 2004, 16, 996 b) S. Raz, O. Testeniere, A. Hecker, S. Weiner, G. Luquet, Biol. Bull. 2002, 203, 269.

[42] W. L. Marshall, J. M. Warakomski, Geochim. Cosmochim. Acta 1980, 44, 915.

[43] A. Navrotsky, Proc. Natl. Acad. Sci. USA 2004, 101, 12096.

[44] M. A. Larson, J. Garside, Chem. Eng. Sci. 1986, 41, 1285.

[45] A. Gal, S. Weiner, L. Addadi, J. Am. Chem. Soc. 2010, 132, 13208.

[46] Y. Oaki, S. Kajiyama, T. Nishimura, H. Imai, T. Kato, Adv. Mater. 2008, 20, 3633.

[47] C. G. Sinn, R. Dimova, M. Antonietti, Macromolecules 2004, 37, 3444.

[48] P. J. Flory, J. Am. Chem. Soc. 1936, 58, 1877.

[49] J. G. Kirkwood, J. Riseman, in Rheology: theory and applications (Ed: F. Eirich), Academic Press, New York 1956, pp. 495.

[50] S. Posner, F. Betts, Acc. Chem. Res. 1975, 8, 273.

[51] a) Y. C. Chang, A. S. Myerson, AlChEJ. 1985, 31, 890; b) R. Mohan, O. Kaytancioglu, A. S. Myerson, J. Cryst. Growth 2000, 217, 393.

[52] D. Horn, J. Rieger, Angew. Chem. Int. Ed. 2001, 40, 4330.

[53] A. Dey, P. H. H. Bomans, F. A. Müller, J. Will, P. M. Frederik, G. de With, N. A. J. M. Sommerdijk, Nat. Mater. 2010, 9, 1010.

[54] a) X. Yang, L. Wang, Y. Qin, Z. Sun, Z. J. Henneman, J. Moradian-Oldak, G. H. Nancollas, J. Phys. Chem. B 2010, 114, 2293; b) P. A. Fang, J. F. Conway, H. C. Margolis, J. P. Simmer, E. Beniash, Proc. Natl. Acad. Sci. USA 2011, 108, 14097. 